\begin{tabular}{|c|c|c|}
\hline \multirow{3}{*}{$\begin{array}{r}\text { Case Reports in } \\
\text { Gastroenterology }\end{array}$} & \multirow{2}{*}{\multicolumn{2}{|c|}{ Case Rep Gastroenterol 2016;10:440-445 }} \\
\hline & & \\
\hline & $\begin{array}{l}\text { DOI: 10.1159/000448067 } \\
\text { Publisnea onine. August 12, } 2016\end{array}$ & $\begin{array}{l}\text { (C) } 2016 \text { The Author(s) } \\
\text { Published by S. Karger AG, Basel } \\
\text { www.karger.com/crg }\end{array}$ \\
\hline & \multicolumn{2}{|c|}{$\begin{array}{l}\text { This article is licensed under the Creative Commons Attribution-NonCommercial } 4.0 \\
\text { International License (CC BY-NC) (http://www.karger.com/Services/OpenAccessLicense) } \\
\text { Usage and distribution for commercial purposes requires written permission. }\end{array}$} \\
\hline
\end{tabular}

\title{
Hepatic Angiosarcoma Associated with Esophageal Variceal Hemorrhage
}

\author{
Zensho Ito $^{a} \quad$ Mikio Kajihara $^{a}$ Yasunobu Kobayashi ${ }^{a} \quad$ Tomoya Kanai $^{\mathrm{a}}$ \\ Yoshihiro Matsumoto ${ }^{a}$ Kazuki Takakura ${ }^{a}$ Toyokazu Yukawa $^{a}$ \\ Toshifumi Ohkusa $^{a}$ Seita Koyama ${ }^{b}$ Hiroo Imazu $^{b}$ Hiroshi Arakawa $^{b}$ \\ Mitsuru Ohata ${ }^{c}$ Shigeo Koido ${ }^{a}$ \\ aDepartment of Gastroenterology and Hepatology, The Jikei University Kashiwa Hospital, \\ Kashiwa, Japan; ${ }^{b}$ Department of Gastrointestinal Endoscopy, The Jikei University Kashiwa \\ Hospital, Kashiwa, Japan; 'Department of Internal Medicine, Hiranuma Clinic, \\ Yokohama, Japan
}

\section{Keywords}

Hepatic angiosarcoma · Esophageal variceal hemorrhage · Endoscopic injection sclerotherapy

\section{Abstract}

Primary hepatic angiosarcoma is a very rare malignancy with a poor prognosis. Because patients present with no specific symptoms, the cancer can grow undetected and most cases are diagnosed too late for resection. We present the case of a 78-year-old Japanese man admitted to our hospital with massive hematemesis and melena. A total gastrectomy had previously been performed on the patient to treat gastric cancer. Endoscopic injection sclerotherapy was performed to control the bleeding from varices over the anastomosis. Computed tomography revealed the presence of multiple atypical liver nodules in the enhanced image. Histological diagnosis of hepatic angiosarcoma was obtained by percutaneous ultrasound-guided liver biopsy. To our knowledge, this is the first report of a patient with hepatic angiosarcoma and acute variceal hemorrhage.

(C) 2016 The Author(s)

Published by S. Karger AG, Basel 


\section{Introduction}

Hepatic angiosarcoma (HA) is a primary hepatic neoplasm that develops from endothelial cells in the liver. Though it is the most common primary liver sarcoma, it has a relatively rare occurrence, accounting for approximately $2 \%$ of primary liver tumors [1]. Some of the risk factors for the development of angiosarcoma include exposure to thorium dioxide (Thorotrast), diethylstilbestrol, vinyl chloride, and arsenic, the use of androgenic steroids, hemochromatosis, von Recklinghausen's disease, and a history of radiation exposure [2], but the etiology is unknown in most cases. Patients with HA primarily present with generalized and nonspecific symptoms, and radiological techniques are limited in the evaluation of HA, so a definitive diagnosis often requires histopathological examination [3]. Reported herein is a case of biopsy-confirmed primary HA, which presented with acute variceal bleeding.

\section{Case Report}

A 78-year-old Japanese man was referred and admitted to our center for hematemesis and melena. He reported a previous history of gastric cancer, for which he had undergone a total gastrectomy at the age of 59 years, but otherwise his past medical history was unremarkable. The patient said that he had long been a habitual drinker (about $50 \mathrm{~g} \mathrm{EtOH}$ nightly for nearly 40 years), but reported no significant history of exposure to carcinogenic chemicals.

On admission, the patient exhibited hepatomegaly which was slightly painful to the touch. Hematological tests revealed that he was slightly anemic $(10.1 \mathrm{~g} / \mathrm{dl})$, with a platelet count of $72 \times 10^{3} / \mu$ l. Laboratory results also demonstrated alterations in liver function: total bilirubin was $1.6 \mathrm{mg} / \mathrm{dl}$ (reference range 0.2-1.0); aspartate aminotransferase was $59 \mathrm{U} / \mathrm{l}$ (reference 10-33); alanine aminotransferase was 43 IU/l (reference 6-35); alkaline phosphatase was $861 \mathrm{U} / \mathrm{l}$ (reference 96-300); serum albumin was $3.1 \mathrm{~g} / \mathrm{dl}$ (reference 3.5-5.2), and prothrombin time (international normalized ratio) was 1.23. The serum levels of tumor markers, e.g., alpha-fetoprotein, carcinoembryonic antigen and carbohydrate antigen 19-9, were within normal values. The serum levels of viral markers such as hepatitis B surface antigen and hepatitis $\mathrm{C}$ virus antibody were also negative.

Computed tomography (CT) images revealed hepatomegaly with multiple hypoattenuating micronodular tumors in both hepatic lobes, which showed heterogeneous enhancement on contrast-enhanced CT (fig. 1). Hepatofugal flow was demonstrated via Doppler ultrasonography (US), suggesting the presence of portal hypertension. Neither splenomegaly nor ascites were observed in the patient.

Initial upper gastrointestinal endoscopy revealed a red polypoid mass covered with white exudate over the anastomosis. Although the mass was not actively bleeding, endoscopic US demonstrated that the mass was associated with the esophageal varix, suggesting that it resulted from a recent variceal hemorrhage (fig. 2a).

On the 7th day in the hospital, the patient presented with melena and an emergency endoscopy demonstrated active bleeding from the lesion (fig. 2b). Endoscopic injection sclerotherapy was promptly performed and successfully controlled the bleeding. A fiberscopeattached balloon was first applied for balloon tamponade, and intra-variceal sclerotherapy followed by para-variceal mucosal sclerotherapy were performed. Posthemostatic reexamination confirmed that there was no recurrence of bleeding, and the polypoid mass had shrunk (fig. 2c). 
With hemostasis assured, histopathological specimens were obtained from the nodular liver for further confirmation using US-guided percutaneous biopsy. The results of the pathological examination revealed malignant proliferation characterized by intrasinusoidal infiltration. The tumor cells were spindle-shaped with marked pleomorphism (fig. 3a) and specific strong immunoreactivity against vascular endothelial markers (CD31 and CD34) (fig. 3b, c).

Due to a steady deterioration in liver function and a worsening health status, the patient was not considered a candidate for chemotherapy or surgical treatment and was transitioned to palliative care. The patient eventually passed away 45 days after hospital admission. A postmortem examination was turned down by his next of kin.

\section{Discussion}

HA is a very rare and aggressive malignant tumor that accounts for a mere $2 \%$ of all primary hepatic malignancies $[1,4]$. Epidemiologically, it is associated with exposure to chemicals such as polyvinyl chloride, Thorotrast and arsenic [2], but HA can develop in the absence of an underlying or pre-existing liver disease and no obvious risk factors have been identified [5]. In the present case, the patient reported no significant history of exposure to the aforementioned chemicals. The patient did, however, describe a history of alcohol consumption, which may have contributed to the development of the disease. Reports have indicated that some cases of HA may be associated with alcoholic cirrhosis [6]. The patient had not been previously diagnosed as cirrhotic and the imaging results showed a lack of splenomegaly, implying the absence of liver cirrhosis.

The clinical diagnosis of HA is complicated and often delayed due to a relative absence of early signs or symptoms. The patient usually presents with nonspecific symptoms such as fatigue, weight loss and abdominal discomfort, followed by ascites, icterus and hepatomegaly. In the present case, however, the patient presented with hematemesis and melena. Literature describing upper gastrointestinal bleeding as the initial presentation of HA is scarce. To our knowledge, noncirrhotic portal hypertension causing acute variceal hemorrhage secondary to HA has actually never been reported. Its exact mechanism is unclear and could be multifactorial. In our case, micronodular tumors were diffusely distributed throughout the hepatic parenchyma. Intrasinusoidal infiltration, severe sinusoidal obstruction and venular occlusion could all have contributed to portal congestion and acute variceal hemorrhage via presinusoidal portal hypertension [7]. The current patient had also previously undergone a total gastrectomy. Previous reports have indicated that altered hyperhemodynamics in such patients could aggravate esophageal varices. The elimination of venous collaterals around the anastomotic area could have weakened available draining veins that were originally decompressing a hypertensive portal system [8]. The patient's alcohol consumption could have also affected the formation of varices; habitual ethanol consumption disturbs hepatic microcirculation and contributes to an altered sinusoidal tone and a subsequent increase in portal pressure, regardless of the presence of established liver cirrhosis [7]. The diagnosis of angiosarcomas via imaging studies is challenging because HA exhibits varied enhancement patterns on multiphasic contrast-enhanced CT. HA can present as an inconclusive multifocal or focal hepatic mass with broad differential diagnoses such as liver abscess, hepatocellular cancer, hepatic metastasis, primary hepatic lymphoma, hepatic cystadenocarcinoma, hemangioblastoma and hepatic epithelioid hemangioendothelioma [9]. 
A pathological diagnosis of HA is therefore necessary. Liver biopsy, either percutaneous or transjugular, is often required in patients with reasonable performance status. Percutaneous biopsy has been shown to be more effective in the diagnosis of HA, while the transjugular approach has been reported to be associated with fewer complications from intra-abdominal hemorrhage [10]. Our patient underwent carefully maneuvered US-guided percutaneous biopsy without complications, and the resulting morphological and immunohistochemical findings led to the diagnosis of HA.

Even with an established diagnosis, treatment of the tumor has yet to be well defined and is largely empirical. HA also exhibits an ominous clinical course with minimal response to treatment; the median time of survival after diagnosis is approximately 6 months, with death resulting from hepatic failure in approximately 50\% of patients and death from hemoperitoneum occurring in approximately $25 \%$ of patients $[5,11]$. When the tumor is confined exclusively to one lobe of the liver, partial hepatectomy is indicated [12]. However, in most cases, including the present one, the tumors have already multiplied and have diffusely infiltrated the liver at the time of diagnosis. The use of chemotherapy has shown only limited improvement in survival rates, and no single treatment regimen has been established $[13,14]$. Targeted therapies such as bevacizumab and sorafenib have been shown to exert limited efficacy [15]. We considered using palliative chemotherapy in the current patient, but the rapid progression of the disease and the steady deterioration in liver function were not conducive to this treatment strategy.

In conclusion, this is the first report of a case of HA with an initial presentation of noncirrhotic portal hypertension resulting in acute variceal hemorrhage. Liver biopsy enabled the definitive diagnosis of HA, but because the malignancy was discovered at a very advanced stage and the patient exhibited a compromised physical status, the patient received no specific oncological treatment and was treated instead with palliative care. The rarity of this disease makes research on it difficult, but further studies on early diagnosis and new effective systemic or local therapies are required to improve prognosis.

\section{Statement of Ethics}

The authors have no ethical conflicts to disclose.

\section{Disclosure Statement}

All authors declare that they have no conflicts of interest relevant to this paper.

\section{References}

1 Molina E, Hernandez A: Clinical manifestations of primary hepatic angiosarcoma. Dig Dis Sci 2003;48:677-682.

-2 Bolt HM: Vinyl chloride-a classical industrial toxicant of new interest. Crit Rev Toxicol 2005;35:307323.

-3 Lyburn ID, Torreggiani WC, Harris AC, Zwirewich CV, Buckley AR, Davis JE, Chung SW, et al: Hepatic epithelioid hemangioendothelioma: Sonographic, CT, and MR imaging appearances. AJR Am J Roentgenol 2003;180:1359-1364.

4 Budd GT: Management of angiosarcoma. Curr Oncol Rep 2002;4:515-519.

5 Zheng YW, Zhang XW, Zhang JL, Hui ZZ, Du WJ, Li RM, Ren XB: Primary hepatic angiosarcoma and potential treatment options. J Gastroenterol Hepatol 2014;29:906-911. 
Ito et al.: Hepatic Angiosarcoma Associated with Esophageal Variceal Hemorrhage

6 Forbes A, Portmann B, Johnson P, Williams R: Hepatic sarcomas in adults: a review of 25 cases. Gut 1987;28:668-674.

7 Sarin SK, Khanna R: Non-cirrhotic portal hypertension. Clin Liver Dis 2014;18:451-476.

-8 Boku M, Sugimoto K, Nakamura T, Kita Y, Zamora CA, Sugimura K: Percutaneous trans-hepatic obliteration for bleeding esophagojejunal varices after total gastrectomy and esophagojejunostomy. Cardiovasc Intervent Radiol 2006;29:1152-1155.

-9 Yang KF, Leow VM, Hasnan MN, Manisekar KS: Primary hepatic angiosarcoma: Difficulty in clinical, radiological, and pathological diagnosis. Med J Malaysia 2012;67:127-128.

10 Rademaker J, Widjaja A, Galanski M: Hepatic hemangiosarcoma: imaging findings and differential diagnosis. Eur Radiol 2000;10:129-133.

11 Bioulac-Sage P, Laumonier H, Laurent C, Blanc JF, Balabaud C: Benign and malignant vascular tumors of the liver in adults. Semin Liver Dis 2008;28:302-314.

12 Weitz J, Klimstra DS, Cymes K, Jarnagin WR, D’Angelica M, La Quaglia MP, Fong Y, et al: Management of primary liver sarcomas. Cancer 2007;109:1391-1396.

13 Abraham JA, Hornicek FJ, Kaufman AM, Harmon DC, Springfield DS, Raskin KA, Mankin HJ, et al: Treatment and outcome of 82 patients with angiosarcoma. Ann Surg Oncol 2007;14:1953-1967.

14 Mathew P, Vakar-Lopez F, Troncoso P: Protracted remission of metastatic epithelioid angiosarcoma with weekly infusion of doxorubicin, paclitaxel, and cisplatin. Lancet Oncol 2006;7:92-93.

15 D'Adamo DR, Anderson SE, Albritton K, Yamada J, Riedel E, Scheu K, Schwartz GK, et al: Phase II study of doxorubicin and bevacizumab for patients with metastatic soft-tissue sarcomas. J Clin Oncol 2005;23:7135-7142.
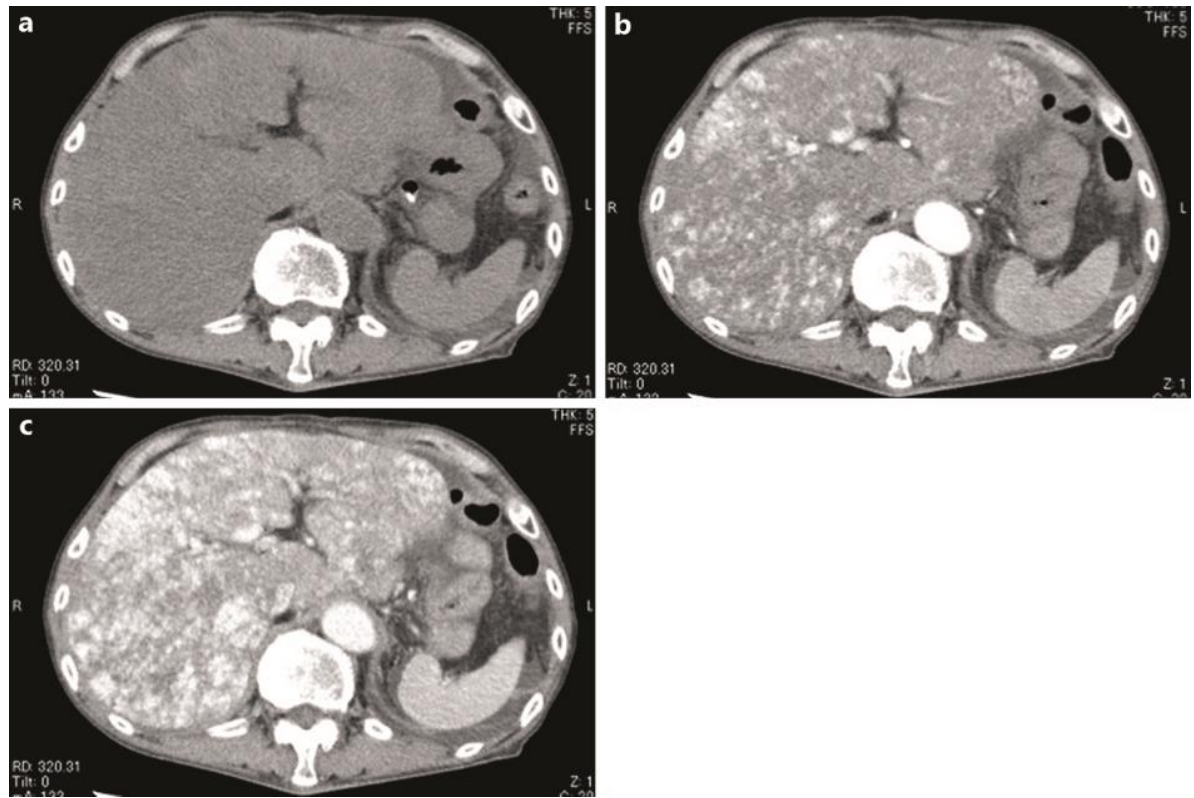

Fig. 1. CT images revealed hepatomegaly with multiple tumors in both hepatic lobes (a), which demonstrated heterogeneous enhancement on contrast-enhanced images (b, c). 

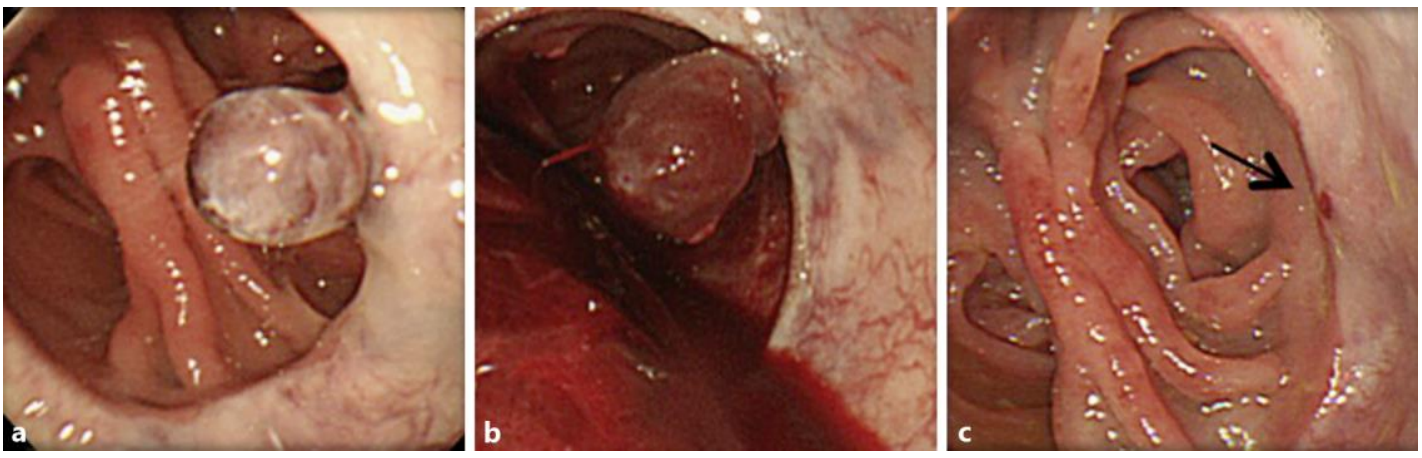

Fig. 2. Initial gastrointestinal endoscopy revealed a red-colored polypoid mass (a), which later exhibited active hemorrhage (b). After endoscopic sclerotherapy, the polypoid mass had shrunk (c; arrow).
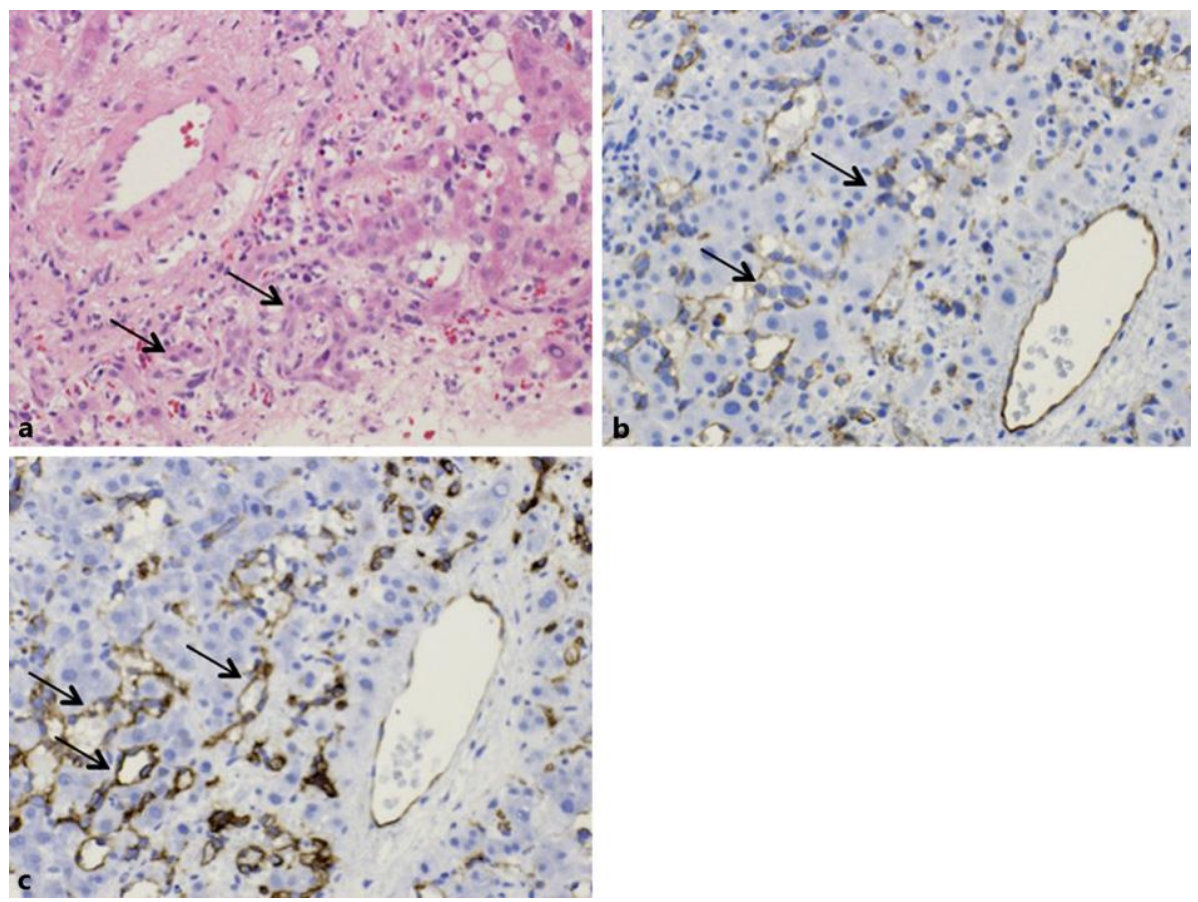

Fig. 3. HE staining showed tumor cells that were spindle-shaped with marked pleomorphism (a). The tumor cells exhibited strong immunoreactivity (arrows) for the specific vascular endothelial markers CD31 (b) and CD34 (c). Original magnification: $\times 40$. 